\title{
PROPRIEDADES FÍSICAS DA MADEIRA DE Araucaria angustifolia (Bert.) O. Kuntze EM FUNÇÃO DA POSIÇÃO NO FUSTE PARA DIFERENTES IDADES
}

\author{
Éverton Hillig ${ }^{1}$, Gilmara de Oliveira Machado², Diego Leonardo Holk³, Giordano Marques Corradi ${ }^{3}$
}

(recebido: 3 de fevereiro de 2010; aceito: 22 de dezembro de 2011)

\begin{abstract}
RESUMO: Conduziu-se este trabalho, com o objetivo de estudar as propriedades físicas da madeira de Araucária (Araucaria angustifolia). Determinaram-se a massa específica aparente e a estabilidade dimensional de árvores de diferentes idades e em diferentes posições ao longo do fuste. A análise dos dados foi realizada em função da idade e da altura no fuste da árvore, além de terem sido relacionadas entre si e com outras variáveis dendrométricas. Verificou-se que houve variação da massa específica entre as idades estudadas, mas não foi possível determinar um padrão de variação. Ocorre uma variação de massa específica ao longo do fuste, sendo que decresce com a altura para todas as idades. A madeira obtida da parte inferior do fuste foi mais instável dimensionalmente. São apresentadas equações lineares da soma das contrações radial e tangencial em função da massa específica aparente. As equações mostraram que mais de $40 \%$ da contração foi explicada pela variação da massa específica aparente.
\end{abstract}

Palavras-chave: Estabilidade dimensional, massa específica, caracterização da madeira.

\section{PHYSICAL PROPERTIES OF WOOD FROM Araucaria angustifolia (Bert.) O. Kuntze AS A FUNCTION OF POSITION IN THE STEM AT DIFFERENT AGES}

\begin{abstract}
The objective of this work was to study the physical properties of wood from Araucaria (Araucaria angustifolia). Parameters included apparent density and dimensional stability of trees at different ages and at different positions along the stem. Data were analyzed as a function of age and height along the tree stem, and then correlated with each other and with other dendrometric variables. A variation was observed in the apparent density between ages, yet it was not possible to determine a variation pattern. A variation was found in the apparent density along the stem, decreasing with increasing heights for all ages. Wood obtained from the lower portion of the stem was more unstable dimensionally. Linear equations are provided of the sum of radial and tangential shrinkage as a function of the apparent density. Equations reveal that more than $40 \%$ of the shrinkage was explained by the variation in apparent density.
\end{abstract}

Key words: Dimensional stability, apparent density, wood characterization.

\section{INTRODUÇÃO}

A Araucaria angustifolia, também conhecida como Pinheiro-do-Paraná, é uma espécie que foi explorada primeiramente nas áreas de sua ocorrência natural, tendo no passado grande importância econômica na região sul do Brasil, fato que levou à sua inclusão na lista de espécies ameaçadas de extinção. Segundo Aquino (2005), houve um intenso processo de exploração predatória dessa espécie, a partir do início do século XIX, levando as reservas naturais de sua ocorrência a menos de $3 \%$ de sua área original.

Embora, num primeiro momento, algumas empresas tenham iniciado reflorestamentos com a araucária, a introdução no país de coníferas exóticas, como o Pinus elliottii e o Pinus taeda, levou essas empresas a substituírem seus plantios por essas espécies. De modo geral, os melhores incrementos e as baixas exigências de solos (CELSO, 2006) foram fatores suficientemente fortes para justificar essa substituição.

Nesse contexto, diversos estudos com araucária foram realizados nas diferentes áreas da ciência florestal e da botânica. Estudos sobre as propriedades da madeira foram realizados na década de 70 e início da década de 80 , especialmente com árvores de ocorrência natural e com árvores jovens de plantios.

Rolim e Ferreira (1974) comentaram que as características da madeira produzida em povoamentos implantados diferem daquela produzida em plantações naturais. Sob espaçamentos amplos há a tendência das coníferas em apresentar anéis de crescimento mais largos, originando madeira de baixa densidade, principalmente nos primeiros anéis de crescimento próximo à medula da árvore.

\footnotetext{
${ }^{1}$ Engenheiro Florestal, Professor Doutor em Engenharia Florestal - Departamento de Engenharia Florestal - UNICENTRO - PR 153, Km 07, Riozinho - 84500-000 - Irati, PR, Brasil - hillig@hotmail.com

${ }^{2}$ Química, Professora Doutora em Ciências e Engenharia de Materiais - Departamento de Engenharia Florestal - UNICENTRO - PR 153, Km 07, Riozinho - 84500-000 - Irati, PR, Brasil - gilmaramachado@yahoo.com.br

${ }^{3}$ Graduando em Engenharia Florestal - Departamento de Engenharia Florestal - UNICENTRO - PR 153, Km 07, Riozinho - 84500-000 - Irati, PR, Brasil-diegoholk@hotmail.com, giordanomcorradi@gmail.com
}

Cerne, Lavras, v. 18, n. 2, p. 257-263, abr./jun. 2012 
Os mesmos autores concluíram que a massa específica básica de Araucaria angustifolia (Bert.) O. Kuntze, de plantações artificiais até 15 anos de idade, cresce no sentido medula-casca, sendo esse crescimento acentuado até nove anos e menos pronunciado a partir dessa idade. Os valores médios de massa específica básica variaram de 0,371 a $0,521 \mathrm{~g} / \mathrm{cm}^{3}$.

Amaral et al. (1971) verificaram que a massa específica básica em árvores de araucária cresceu da medula para a casca. Esse crescimento foi bastante acentuado na madeira mais próxima à medula, ou seja, madeira juvenil, enquanto que na madeira mais próxima à casca (madeira adulta) ele foi pequeno, com uma tendência para se tornar constante.

Oliveira e Tomaselli (1981), determinaram os valores de contração na largura e na espessura de peças de madeira obtidas de árvores com 15 anos de idade, estimando a contração tangencial em 3,56\% e a contração radial em 2,37\%. Ressalta-se que esses valores de contração se referem à secagem até a umidade de equilíbrio (UE), representando, assim, pouco mais da metade da contração total da madeira (PSF até a $0 \%$ de umidade).

Mais recentemente a espécie voltou a despertar interesse, por vários motivos. As condições de processamento é uma vantagem que pode ser apontada. Segundo Hillig et al. (2006), a geração de resíduos no desdobro e beneficiamento da madeira, em serrarias da região do Corede-Serra do Rio Grande do Sul, apresentou média de 27\% para a Araucaria angustifolia, 36\% para o Eucalyptus spp. e 37\% para o Pinus spp. Em outro estudo, de quantificação de resíduos e rendimento no desdobro, concluiu-se que, em média, o processo de desdobro de toras de Araucaria angustifolia apresenta rendimento superior quando comparado com outras espécies encontradas na literatura (VALÉRIO et al., 2007).

$\mathrm{Na}$ área de Propriedades da Madeira, Santini et al. (2000) determinaram as propriedades físicas e mecânicas da madeira de araucária proveniente de floresta plantada. Os autores encontraram, para madeira de araucária de 19 anos, um coeficiente de contração volumétrica de 10,4\% e uma massa específica básica de $0,40 \mathrm{~g} / \mathrm{cm}^{3}$.

Mattos et al. (2006) encontraram valor médio de massa específica básica igual a $0,425 \mathrm{~g} / \mathrm{cm}^{3}$, para madeira de araucária proveniente de árvores de 38 anos de idade obtidas em um talhão experimental plantado em Rio Negro, Paraná.

Em um estudo sobre a qualidade macroscópica da madeira de Pinus elliottii (13 anos), Pinus taeda (13 anos) e Araucaria angustifolia (19 anos) oriundas de reflorestamentos jovens, Barboza et al. (2007) verificaram que a madeira de araucária mostra-se superior ao gênero Pinus, quando se avalia a qualificação da madeira pela característica dos nós e pelo número de anéis por polegada. No entanto, os autores concluíram que o parâmetro "porcentagem de lenho tardio" não foi eficiente para qualificação da madeira de araucária.

Melo et al. (2007) observaram que a massa específica e o número de anéis por centímetro quadrado para a madeira de Araucaria angustifolia variaram nas diferentes regiões da árvore, demonstrando variação linear crescente da medula para casca para os dois parâmetros.

Barboza et al. (2008) verificaram que a massa específica básica da madeira de araucária de árvores com 19 anos de idade apresentou significativa diferença em relação às posições ao longo do fuste. A massa especifica básica média encontrada foi de $0,40 \mathrm{~g} / \mathrm{cm}^{3}$, diminuindo proporcionalmente com o aumento da altura comercial.

Mattos et al. (2009) encontraram uma contração volumétrica de $10,41 \%$ e um coeficiente de anisotropia igual a 1,41 para madeira de araucária. Os autores concluíram que a contração volumétrica da espécie apresentou menor variação com o aumento da massa específica básica quando comparada com Pinus elliottii e Pinus taeda. Nesse estudo, a equação de regressão da contração volumétrica em função da massa específica básica apresentou coeficiente de determinação igual a 0,07.

Por outro lado, não foram encontrados estudos que avaliem a variação da massa específica da espécie para árvores de diferentes idades. Sendo assim, neste trabalho, objetivou-se determinar as propriedades físicas da madeira de Arancaria angustifolia (Bert.) O. Kuntze, de ocorrência na floresta Ombrófila Mista, da Região Centro-Oeste do Paraná, verificando sua variação entre árvores de diferentes idades e nas diferentes posições ao longo do fuste.

\section{MATERIAL E MÉTODOS}

\subsection{Amostragem}

Foram utilizados discos com cinco centímetros de espessura de sete exemplares com 32, 33, 43, 49, 49, 53 e 58 anos de idade, provenientes da FLONA de IratiPR, anteriormente usados para Análise de Tronco. Foram analisados os discos a 25, 50, 75 e $95 \%$ da altura total, mais um disco a $0,1 \mathrm{~m}$ (base) e outro na altura do peito (DAP). De cada disco, foram retirados quatro corpos-de-prova em forma de cunha, opostos entre si, dois para determinação da massa especifica aparente e dois para determinação da estabilidade dimensional (Figura 1). Na caracterização do disco, foi utilizado o valor médio das medições em cunhas opostas.

Cerne, Lavras, v. 18, n. 2, p. 257-263, abr./jun. 2012 


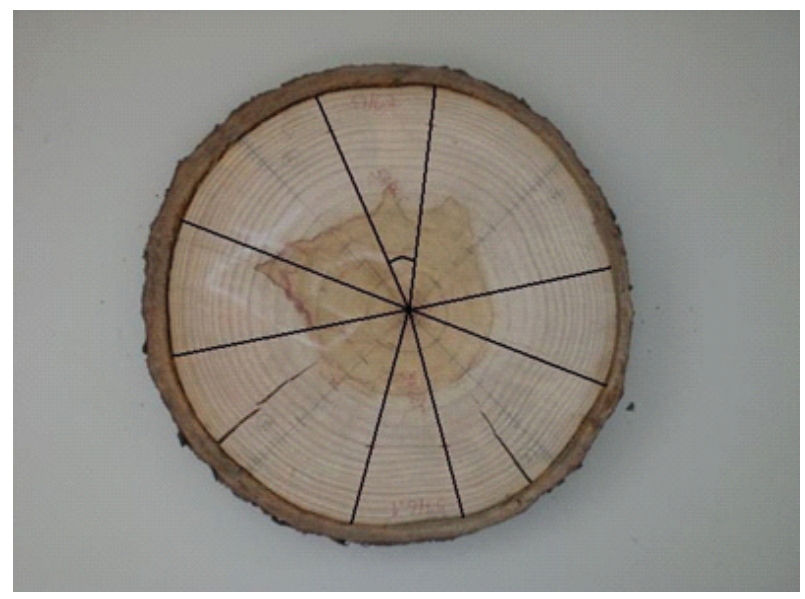

Figura 1 - Esquema de corte dos corpos-de-prova.

Figure 1 - Illustration of specimen sections.

\subsection{Determinação da massa específica aparente}

A massa específica de cada corpo-de-prova foi determinada pelo método da balança hidrostática, seguindo as orientações da norma NBR 7190 para estruturas de madeira (ASSOCIAÇÃO BRASILEIRA DE NORMAS TÉCNICAS - ABNT, 1997), adaptada. Para cálculo da massa específica foi utilizada a equação 1 , sendo que foi usada a massa seca e o volume ao teor de umidade de equilíbrio (aproximadamente 12,7\%). Esse tipo de massa específica aparente foi adotado, objetivando diminuir a influência da água na massa específica pelo uso de massa seca.

$m e=\frac{m}{v}$

onde: $\mathrm{me}=$ massa específica aparente

$\mathrm{m}=$ massa seca

$\mathrm{v}=$ volume ao teor de umidade de equilíbrio

\subsection{Determinação da estabilidade dimensional}

Para a análise da estabilidade dimensional, das cunhas foram retirados corpos-de-prova retangulares. Seguindo a metodologia adaptada de Rezende et al. (1995), para cada disco coletado preparou-se uma amostra com dimensões variáveis, em função do tamanho do disco, e com a aresta lateral maior na direção radial. Essas amostras foram colocadas submersas em água, para saturação, por um período de 30 dias. Atingida essa condição, as amostras foram submetidas a um processo de secagem natural até atingir a umidade de equilíbrio com o ambiente do laboratório, em torno de 12,7 \% (base seca). A partir dessa umidade as amostras foram colocadas em estufa, a temperatura de $103^{\circ} \mathrm{C}$, para a sua completa secagem (teor de umidade de $0 \%$ ). Realizaram-se as medições com os corpos de prova saturados em água, ao teor de umidade de equilíbrio e após secagem em estufa a $103^{\circ} \mathrm{C} \pm 2^{\circ} \mathrm{C}$. A medição dos corpos-de-prova foi realizada em quatro pontos: dois na direção tangencial e dois na direção radial, com paquímetro, tendo como referência a metade da espessura do corpo-deprova e marcados como ilustrado na Figura 2.

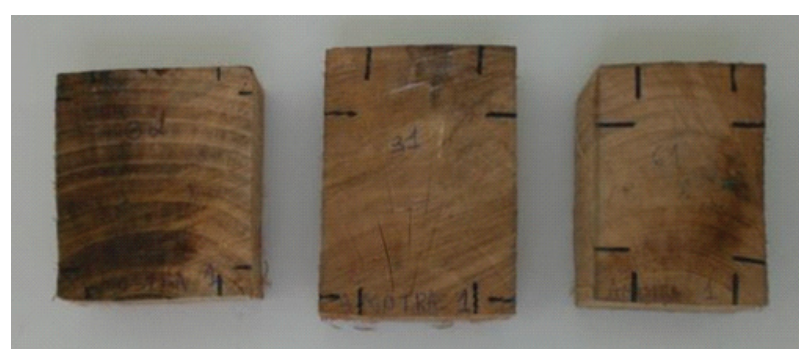

Figura 2 - Corpos-de-prova com marcação dos pontos de medição para determinação das contrações radial e tangencial.

Figure 2 - Specimens marked with measuring points for determination of radial and tangential shrinkage.

Com os dados obtidos, determinaram-se as contrações radial e tangencial, que somadas podem ser consideradas como equivalentes à contração volumétrica. As expressões de cálculo se encontram nas equações 2 , 3 e 4 .

$C R=\frac{\text { Medida saturada } D R-\text { Medida seca } D R}{\text { Medida saturada } D R}$
$C T=\frac{\text { Medida saturada DT }- \text { Medida seca } D T}{\text { Medida saturada DT }}$

$C V \cong C R+C T$

onde: $\mathrm{CR}=$ Contração radial

$\mathrm{CT}=$ Contração tangencial

$\mathrm{CV}=$ Contração volumétrica

$\mathrm{DR}=$ Direção radial

DT = Direção tangencial

\subsection{Análise dos dados}

A análise dos dados de massa específica aparente e de contração foi realizada em função da idade e da altura no fuste da árvore, além de terem sido relacionadas entre si e com outras variáveis dendrométricas: DAP, altura total da árvore e densidade média ponderada da árvore.

Cerne, Lavras, v. 18, n. 2, p. 257-263, abr./jun. 2012 
Primeiramente foram realizados os testes de homogeneidade da variância e de normalidade. Estando confirmadas essas prerrogativas, procedeu-se a análise de variância para cada idade e para cada altura relativa na árvore. Nessa análise, as duas primeiras alturas são absolutas: 0,1 e 1,3 metros, enquanto as outras quatro são relativas: $25,50,75$ e $95 \%$ da altura total.

Após, as quatro alturas relativas foram transformadas em absolutas para correlação com a massa específica e a contração. Com base nessa correlação, foi realizada análise por regressão linear. Todos os testes estatísticos foram realizados com software SPSS.

\section{RESULTADOS E DISCUSSÃO}

\subsection{Massa específica aparente e contrações radial e tangencial em função da idade e da altura na árvore}

A análise de variância mostrou haver diferenças significativas entre a massa específica aparente nas diferentes alturas ao longo do fuste e nas diferentes idades estudadas. O mesmo ocorreu para a soma das contrações radial e tangencial $(\mathrm{CR}+\mathrm{CT})$. Por outro lado, a interação entre altura ao longo do fuste e idade da árvore não foi significativa para as duas variáveis estudadas. Na Tabela 1 , apresentam-se as médias de massa específica e de CR + CT para cada altura estudada.

Verifica-se, na Tabela 1, que tanto a massa específica, como a soma das contrações radial e tangencial, diminuem com o aumento da altura na árvore, estando de acordo com os estudos de Barboza et al. (2008). Nas alturas de $75 \%$ e $95 \%$, foi observado que não houve diferença estatística significativa para a média da soma das contrações radial e tangencial, mostrando que nessa altura da árvore houve menor relação entre essa propriedade e a massa específica aparente.

Com relação às médias de massa específica aparente determinadas para as diferentes idades (Tabela 2), não foi possível determinar um padrão de variação. A análise de variância demonstrou haver diferenças entre as médias, mas verificou-se que os maiores valores médios foram da árvore de menor idade (32 anos), não sendo significativamente diferentes das árvores de 49, 53 e 58 anos que são as de maior idade.

Os resultados encontrados para contração $(\mathrm{CR}+\mathrm{CT})$ seguiram a mesma tendência da massa específica aparente, variando de 7,58 a 11,38\% em função da altura na árvore e de 9,27 a 10,74\% em função da idade, estando próximos aos encontrados por Oliveira e Tomaselli (1981) e Santini et al. (2000), para contração volumétrica.

Cerne, Lavras, v. 18, n. 2, p. 257-263, abr./jun. 2012
Tabela 1 - Valores médios de massa específica aparente (me) e da soma das contrações radial e tangencial para cada altura estudada.

Table 1 - Mean values of apparent density (me) and sum of radial and tangential shrinkage for each height.

\begin{tabular}{|c|c|c|}
\hline Altura & me aparente* $\left(\mathrm{g} / \mathrm{cm}^{3}\right)$ & $\mathrm{CR}+\mathrm{CT}^{*}(\%)$ \\
\hline $0,1 \mathrm{~m}$ & $0,592 \mathrm{a}$ & $11,38 \mathrm{a}$ \\
\hline $1,3 \mathrm{~m}$ & $0,554 \mathrm{a}, \mathrm{b}$ & $11,35 \mathrm{a}$ \\
\hline $25 \%$ & $0,501 \quad b, c$ & $10,93 \mathrm{a}, \mathrm{b}$ \\
\hline $50 \%$ & 0,453 & $9,65 \quad b, c$ \\
\hline $75 \%$ & 0,420 & 7,58 \\
\hline $95 \%$ & 0,360 & 8,51 \\
\hline
\end{tabular}

$\mathrm{CR}+\mathrm{CT}=$ Soma das contrações radial e tangencial

*Médias seguidas por mesmas letras não diferem pelo teste de Tukey, ao nível de $5 \%$ de probabilidade de erro.

$C R+C T=$ Sum of radial and tangential shrinkage

*Means followed by the same letters do not differ by the Tukey test, at the $5 \%$ error probability level.

Tabela 2 - Valores médios de massa específica aparente (me) e da soma das contrações radial e tangencial para cada idade estudada.

Table 2-Mean values of apparent density (me) and sum of radial and tangential shrinkage for each age.

\begin{tabular}{ccc}
\hline Idade (anos) & me aparente* $\left(\mathrm{g} / \mathrm{cm}^{3}\right)$ & $\mathrm{CR}+\mathrm{CT}^{*}(\%)$ \\
\hline 32 & $0,528 \mathrm{~b}$ & $10,58 \mathrm{a}, \mathrm{b}$ \\
33 & $0,457 \mathrm{a}$ & $9,84 \mathrm{a}, \mathrm{b}$ \\
43 & $0,459 \mathrm{a}$ & $9,27 \mathrm{a}$ \\
49 & $0,472 \mathrm{a}, \mathrm{b}$ & $9,35 \mathrm{a}, \mathrm{b}$ \\
53 & $0,474 \mathrm{a}, \mathrm{b}$ & $10,15 \mathrm{a}, \mathrm{b}$ \\
58 & $0,498 \mathrm{a}, \mathrm{b}$ & $10,74 \mathrm{~b}$ \\
\hline
\end{tabular}

$\mathrm{CR}+\mathrm{CT}=$ Soma das contrações radial e tangencial

*Médias seguidas por mesmas letras não diferem pelo teste de Tukey, ao nível de 5\% de probabilidade de erro.

$C R+C T=$ Sum of radial and tangential shrinkage

* Means followed by the same letters do not differ by the Tukey test, at the 5\% error probability level.

Na Figura 3, apresenta-se a variação da massa específica da madeira em função da altura na árvore, para cada idade, com as respectivas linhas de regressão. Verificou-se, numa análise estatística, que a interação entre idade e altura não foi significativa, por outro lado o ajuste da linha de regressão apresentou melhor coeficiente de determinação $\left(\mathrm{R}^{2}\right)$ quando separado por idade.

$\mathrm{Na}$ Figura 4, apresenta-se a variação da soma das contrações radial e tangencial em função da altura na árvore para cada idade. Verifica-se que segue o mesmo padrão da massa específica aparente, mas com coeficientes de determinação $\left(\mathrm{R}^{2}\right)$ inferiores. 


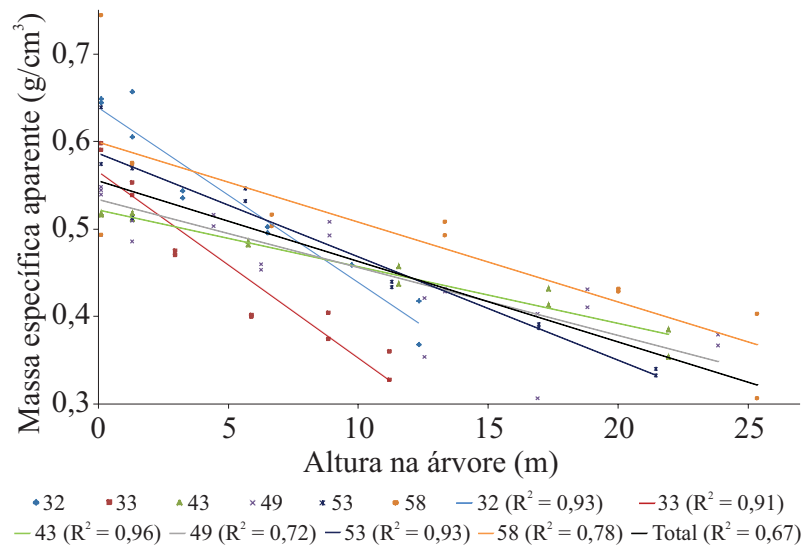

Figura 3 - Massa específica aparente em função da altura na árvore, para cada idade, com respectivas linhas de regressão (Coeficiente de Determinação - $\mathrm{R}^{2}$ entre parênteses).

Figure 3 - Apparent density as a function of height position, for each age, with the respective regression lines (Coefficient of Determination $-R^{2}$ in brackets).

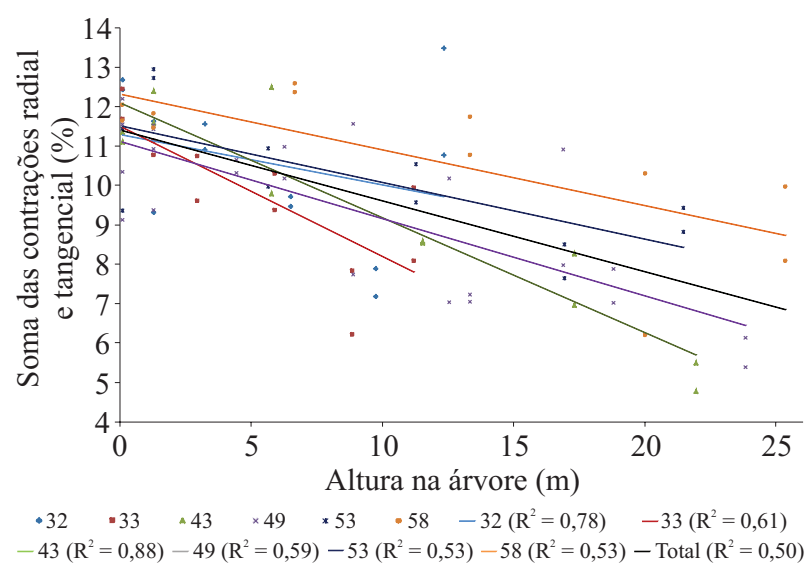

Figura 4 - Soma das Contrações Radial e Tangencial em função da altura na árvore, para cada idade, com respectivas linhas de regressão (Coeficiente de Determinação - $\mathrm{R}^{2}$ entre parênteses).

Figure 4-Sum of radial and tangential shrinkage as a function of height position, for each age, with the respective regression lines (Coefficient of Determination $-R^{2}$ in brackets).

\subsection{Massa específica aparente e contrações médias de cada árvore}

Para melhor entender o fato de a idade de 32 anos ter apresentado maior massa específica que as outras idades e também na tentativa de obter uma média mais representativa, calculou-se a massa específica média ponderada, em função do volume de cada seção entre os discos, para cada árvore. Os valores são apresentados na
Tabela 3, juntamente com as contrações médias ponderadas e as outras variáveis dendrométricas.

Verificou-se que a massa específica aparente média ponderada de cada árvore seguiu o mesmo padrão da massa específica aparente média de cada idade, ou seja, a média aritmética foi semelhante à média ponderada. Isso em razão de terem sido retirados os discos de duas alturas absolutas $(0,1 \mathrm{e}$ 1,3 metros) e de alturas relativas da árvore, a partir de $25 \%$ da altura total e até a extensão do fuste. Assim, embora as toras inferiores tenham maior diâmetro, não têm, necessariamente, maior volume, pois têm menor comprimento.

As contrações radial e tangencial, por outro lado, apresentam dados um pouco diferentes, destacando que a árvore 12, de maior massa específica, apresentou uma soma das contrações radial e tangencial intermediária e a árvore cinco, de 49 anos, apresentou a menor contração, seguida da árvore 17 de mesma idade.

$\mathrm{O}$ fato de a árvore 12 apresentar maior massa específica que a árvore 13, de idade semelhante, poderia sugerir que a árvore 13 obteve maior crescimento e, por isso, menor número de anéis por polegada. No entanto, observa-se, na Tabela 3, que o diâmetro e a altura da árvore 12 são maiores que da árvore 13 .

Barboza et al. (2007) comentam que a classificação da madeira de araucária pelo número de anéis por polegada e pela porcentagem de lenho tardio pode não ser adequada, pois se trata de um critério usado para classificação de madeira de Pinus. Esse último parâmetro, em especial, não pode ser usado para Araucária, pois a passagem do lenho tardio para o inicial se faz gradativamente tornando difícil uma quantificação. Assim, outros fatores podem ter influenciado a massa específica da árvore 12, como o percentual de lenho tardio, proporção de medula, defeitos naturais, taxa de crescimento e fatores silviculturais, como espaçamento e solo.

\subsection{Estabilidade dimensional em função da massa específica aparente}

Na Tabela 4, são apresentadas as equações, obtidas por regressão linear, da soma das contrações radial e tangencial em função da massa específica aparente, para cada idade e para todas as idades. Nessa tabela, as idades de 32 e 33 anos foram agrupadas como uma só idade. Verifica-se um melhor ajuste da regressão linear, quando se compara o coeficiente de determinação $\left(\mathrm{R}^{2}\right)$ e o erro padrão da estimativa $\left(\mathrm{S}_{\mathrm{yx}}\right)$, separando as idades, exceto para a idade de 49 anos. Verifica-se, também, pelos coeficientes de determinação $\left(\mathrm{R}^{2}\right)$, que, na maior parte das árvores (ou idades), a massa específica explica pouco mais de $40 \%$ da soma das contrações radial e tangencial.

Cerne, Lavras, v. 18, n. 2, p. 257-263, abr./jun. 2012 
Tabela 3 - Valores médios, ponderados por volume da seção na tora, de Massa Específica e de Contração para cada árvore, juntamente com as variáveis dendrométricas.

Table 3 - Mean values, weighted according to volume of log section, of Apparent Density and Shrinkage for each tree, along with the dendrometric variables.

\begin{tabular}{cccccccc}
\hline Árvore & Idade & Altura total $(\mathrm{m})$ & DAP $(\mathrm{cm})$ & $\mathrm{mep}\left(\mathrm{g} / \mathrm{cm}^{3}\right)$ & $\mathrm{CRp}(\%)$ & $\mathrm{CTp}(\%)$ & $(\mathrm{CR}+\mathrm{CT}) \mathrm{p}(\%)$ \\
\hline 5 & 49 & 17,80 & 23,36 & 0,49 & 3,59 & 6,09 & 9,68 \\
12 & 32 & 13,00 & 22,00 & 0,53 & 3,77 & 6,16 & 9,93 \\
13 & 33 & 11,80 & 16,76 & 0,45 & 3,76 & 5,86 & 9,62 \\
17 & 49 & 25,10 & 34,56 & 0,42 & 3,55 & 5,33 & 8,88 \\
31 & 53 & 22,60 & 40,26 & 0,47 & 3,85 & 6,19 & 10,04 \\
36 & 58 & 26,70 & 31,16 & 0,49 & 4,59 & 6,46 & 11,05 \\
38 & 43 & 23,10 & 25,50 & 0,46 & 3,51 & 5,92 & 9,43 \\
\hline
\end{tabular}

mep $=$ massa específica aparente ponderada $\mathrm{CRp}=$ Contração radial ponderada; $\mathrm{CT} p=$ Contração tangencial ponderada . Mep $=$ weighted apparent density; $C R p=$ weighted radial shrinkage; CTp $=$ weighted tangential shrinkage .

Tabela 4 - Equações lineares da Soma das Contrações Radial e Tangencial (CR + CT) em função da Massa Específica Aparente (me), para cada idade e para todas as idades.

Table 4 - Linear equations of the Sum of Radial and Tangential Shrinkage $(C R+C T)$ as a function of the Apparent Density (me), for each age and for all ages.

\begin{tabular}{ccccc}
\hline Idade & Equação & $\mathrm{R}^{2}$ & $\mathrm{~S}_{\mathrm{vx}}$ & $\mathrm{F}$ \\
\hline $32 / 33$ & $\mathrm{CR}+\mathrm{CT}=3,653+12,727 \mathrm{me}$ & 0,492 & 1,30 & $19,38^{*}$ \\
43 & $\mathrm{CR}+\mathrm{CT}=-10,907+43,971 \mathrm{me}$ & 0,878 & 0,96 & $72,26^{*}$ \\
49 & $\mathrm{CR}+\mathrm{CT}=1,074+17,861 \mathrm{me}$ & 0,413 & 1,57 & $15,46^{*}$ \\
53 & $\mathrm{CR}+\mathrm{CT}=5,095+10,656 \mathrm{me}$ & 0,434 & 1,28 & $7,66^{*}$ \\
58 & $\mathrm{CR}+\mathrm{CT}=2,300+17,554 \mathrm{me}$ & 0,514 & 1,39 & $10,57^{*}$ \\
Todas & $\mathrm{CR}+\mathrm{CT}=2,133+16,160 \mathrm{me}$ & 0,448 & 1,50 & $64,84^{*}$ \\
\hline
\end{tabular}

*Significativo ao nível de $5 \%$ de probabilidade de erro

R2 = Coeficiente de Determinação; Syx = Erro padrão da estimativa; F = Fator do teste de variância

*Significant at the 5\% error probability level

$R 2=$ Coefficient of Determination; Syx $=$ Standard error of estimate $;=$ Factor of variance test

\section{CONCLUSÕES}

Para madeira de Araucaria angustifolia, verificouse que houve variação da massa específica entre as idades estudadas, mas não foi possível estabelecer um padrão de variação. Ocorre uma variação da massa específica ao longo do fuste para todas as idades, sendo que decresce com a altura na árvore. Verificou-se, também, que a madeira obtida da parte inferior do fuste foi mais instável dimensionalmente. As equações lineares para estimar a soma das contrações radial e tangencial em função da massa específica aparente da madeira mostraram que, na maior parte das árvores, a variação da massa específica aparente explicou mais de $40 \%$ da contração (CR + CT). A estimativa obtida separadamente por idade mostrou-se um pouco superior à obtida para todas as idades juntas.

\section{AGRADECIMENTOS}

À Fundação Araucária e à Universidade Estadual do Centro-Oeste (UNICENTRO) pelo financiamento das bolsas e dos recursos financeiros necessários. Ao professor Afonso Figueiredo Filho e sua equipe por cederem o material de estudo.

\section{REFERÊNCIAS}

AMARAL, A. C. B.; FERREIRA, M.; BANDEL, G.;

AMARAL, A. C. B. Variação da densidade básica da madeira produzida pela Araucaria angustifolia (Bert.) O. Kuntze no sentido medula-casca em árvores do sexo masculino e feminino. Revista IPEF, Piracicaba, n. 2/3, p. 119-127, 1971.

Cerne, Lavras, v. 18, n. 2, p. 257-263, abr./jun. 2012 
AQUINO, F. M. Cultivo da Araucaria angustifolia: análise de viabilidade econômico-financeira. Florianópolis: BRDE, $2005.53 \mathrm{p}$.

ASSOCIAÇÃO BRASILEIRA DE NORMAS TÉCNICAS. NBR 7190: projetos de estruturas de madeira. Rio de Janeiro, 1997.

BARBOZA, M. F.; GATTO, D. A.; OLIVEIRA, L. S.; MÜLLER, M. T.; CALEGARI, L.; STANGERLIN, D. M.; TREVISAN, R. Qualidade macroscópica da madeira de Pinus elliottii, Pinus taeda e Araucaria angustifolia oriundas de reflorestamentos jovens. In: CONGRESSO DE INICIAÇÃO CIENTÍFICA, 16.; ENCONTRO DE PÓS-GRADUAÇÃO, 9., 2007, Pelotas. Anais... Pelotas: UFPel, 2007. v. 1, p. 1-4.

BARBOZA, M. F.; GATTO, D. A.; STANGERLIN, D. M.; CALEGARI, L.; MELO, R. R.; BELTRAME, R. Variação axial da massa específica básica da madeira de Araucaria angustifolia (Bert.) O. Kuntze oriunda de floresta plantada. In: CONGRESSO FLORESTAL DO RIO GRANDE DO SUL, 10.; SEMINÁRIO MERCOSUL DA CADEIA MADEIRA, 1., 2008, Nova Prata. Anais... Santa Maria: UFSM, 2008. v. 1, p. 110 .

CELSO, B. Análise comparativa da eficiência entre as espécies florestais Pinus taeda e Araucaria angustifolia (Bert.) O. Kuntze no seqüestro de $\mathrm{CO} 2 \mathrm{em}$ reflorestamento na Região Sul do Brasil. 2006. 143 p. Dissertação (Mestrado em Engenharia Ambiental) - Universidade Regional de Blumenau, Blumenau, 2006.

HILLIG, É.; SCHNEIDER, V. E.; WEBER, C.; TECCHIO, R. D. Resíduos de madeira da indústria madeireira: caracterização e aproveitamento. In: ENCONTRO NACIONAL DE ENGENHARIA DE PRODUÇÃO, 26., 2006, Fortaleza.

Anais... Rio de Janeiro: ABEPRO, 2006. p. 1-7.

MATTOS, B. D.; GATTO, D. A.; STANGERLIN, D. M.; MELO, R. R.; BELTRAME, R. Correlação entre massa específica e retratibilidade da madeira de três coníferas da região sul do brasil. In: CONGRESSO DE INICIAÇÃO
CIENTÍFICA, 28.; ENCONTRO DE PÓS-GRADUAÇÃO, 11.; MOSTRA CIENTÍFICA, 1., 2009, Pelotas. Anais... Pelotas: UFPel, 2009. v. 1, p. 1-5.

MATTOS, P. P.; BORTOLI, C.; MARCHESAN, R.; ROSOT, N. C. Caracterização física, química e anatômica da madeira de Araucaria angustifolia (Bert.) O. Kuntze. Colombo: Embrapa, 2006. 4 p. (Comunicado técnico, 160).

MELO, R. R.; STANGERLIN, D. M.; CALEGARI, L.; MÜLLER, M. T.; TREVISAN, R.; GATTO, D. A. Correlação entre massa específica e número de anéis para a madeira de Araucaria angustifolia. In: CONGRESSO NORDESTINO DE ENGENHARIA FLORESTAL, 1., 2007, Recife. Anais... Recife: UFRPE, 2007. v. 1, p. 1-3.

OLIVEIRA, V. S.; TOMASELLI, I. Efeito da secagem a altas temperaturas em algumas propriedades físicas e mecânicas de madeira jovem de Araucararia angustifolia. Floresta, Curitiba, v. 1, n. 12, p. 17-25, 1981.

REZENDE, M. A.; SAGLIETTI, J. R. C.; GUERRINI, I. A. Estudo das interrelações entre massa específica, retratibilidade e umidade da madeira do Pinus caribaea var. hondurensis aos 8 anos de idade. Revista IPEF, Piracicaba, n. 48/49, p. 133$141,1995$.

ROLIM, M. B.; FERREIRA, M. Variação da densidade básica da madeira produzida pela Araucaria angustifolia (Bert.) O. Kuntze em função dos anéis de crescimento. Revista IPEF, Piracicaba, n. 9, p. 47-55, 1974.

SANTINI, E. J.; HASELEIN, C. R.; GATTO, D. A. Análise comparativa das propriedades físicas e mecânicas da madeira de três coníferas de florestas plantadas. Ciência Florestal, Santa Maria, v. 10, n. 1, p. 85-93, 2000.

VALÉRIO, A. F.; WATZLAWICK, L. F.; SANTOS, R. T.; BRANDELERO, C.; KOEHLER, H. S. Quantificação de resíduos e rendimento no desdobro de Araucaria angustifolia (Bert.) O. Kuntze. Floresta, Curitiba, v. 37, p. 387-398, 2007. 